\title{
Reflets
}

Revue ontaroise d'intervention sociale et communautaire

\section{Le service social au Sénégal : recherche d'un sens ou histoire d'une réception}

\section{Lena D. Ndiaye}

Volume 7, numéro 2, automne 2001

Le travail social en Ontario

URI : https://id.erudit.org/iderudit/026365ar

DOI : https://doi.org/10.7202/026365ar

Aller au sommaire du numéro

Éditeur(s)

Reflets : Revue ontaroise d'intervention sociale et communautaire

ISSN

1203-4576 (imprimé)

1712-8498 (numérique)

Découvrir la revue

Citer cet article

Ndiaye, L. D. (2001). Le service social au Sénégal : recherche d'un sens ou

histoire d'une réception. Reflets, 7(2), 172-191. https://doi.org/10.7202/026365ar

Tous droits réservés (C) Reflets : Revue ontaroise d'intervention sociale et communautaire, 2001
Ce document est protégé par la loi sur le droit d'auteur. L'utilisation des services d’Érudit (y compris la reproduction) est assujettie à sa politique d'utilisation que vous pouvez consulter en ligne.

https://apropos.erudit.org/fr/usagers/politique-dutilisation/ 


\section{Le service social au Sénégal : recherche $d$ 'un sens ou histoire d'une réception}

par

Lena. D. Ndiaye,

formateur, École nationale des travailleurs sociaux, Dakar, Sénégal et étudiant à la maîtrise en service social, Université Laurentienne.

\section{Préambule}

L'usage, dans le cadre de cet article, nous recommande d'indiquer avant toutes élucubrations, d'où nous nous exprimons. Quelle position ou fonction, quelle expérience, quel savoir nous autorisent à écrire sur le service social au Sénégal? Travailleur social sénégalais, étudiant à l'Université Laurentienne de Sudbury, le prétexte pour écrire nous a été fourni par le responsable de la revue Reflets et cette occasion offre une prise à la légitimité de notre plume.Voilà d'où nous tenons notre mandat.

Nous ne parlerons pas du Sénégal comme l'un des premiers pays africains à mettre en place des agences sociales parce que beaucoup d'experts nationaux dans le domaine social s'en réclame également. De nombreuses rencontres entre travailleurs sociaux ont servi de tribune pour parler de l'expérience sénégalaise en matière de service social. C'est pourquoi, pour y voir plus clair, nous avons trouvé judicieux de nous arracher à ces lieux communs. Nous n'avons pas voulu nous laisser bercer par le discours ambiant 
pour interroger le champ, car au-delà de ce discours, il y a des hommes et des femmes, pris dans une histoire dont ils n'ont pas la complète maîtrise. Ce détachement nous permet de mieux saisir la situation actuelle du service social au Sénégal et par delà, sa trajectoire historique.

Notre propos se veut une contribution aux tentatives de rendre visibles certains aspects du service social sénégalais. Il s'inscrit dans la quête d'un sens, car comment parler du service social au Sénégal sans faire référence à celui de la métropole, la France. Si le service social s'est construit en Occident à partir d'un référentiel crédible, au Sénégal, il n'est interrogeable que d'un point de vue périphérique. Il s'est opéré au Sénégal une réception manifeste et massive des éléments structurant le service social en France. Si le modèle s'avère inopérant, c'est qu'il s'intègre difficilement aux structures et au contexte sénégalais.

Ainsi, le discours professionnel tarde à émerger malgré le militantisme ardent de certains travailleurs sociaux. Le travail social (jouons à cache à cache avec le concept de service social) est généralement peu ou mal connu à travers les sociétés. Au Sénégal, il n'est connu pratiquement que par ceux qui le pratiquent. Par rapport à d'autres professions acceptées comme le droit, la médecine, le travail social n'existe même pas. La résolution des problèmes sociaux s'est toujours effectuée dans le contexte communautaire de «l'arbre à palabre», «le bois sacré» ou le «bangou diaxlé» (littéralement, «le banc des problèmes»).

La question relative à notre position, à notre fonction, trouve ici toute sa pertinence. En effet, le service social est-il une profession pouvant réclamer une légitimité au Sénégal? Si l'on se réfêre aux critères de Grenwood (cité par Beauvolsk 2001), la reconnaissance d'une profession nécessite une base théorique organisée et solide, une crédibilité académique, une sanction sociale, un code de déontologie, une culture professionnelle. À la lumière de ceux-ci, nous pouvons dire que le service social en Afrique et au Sénégal en particulier, ne s'est pas encore constitué comme une profession à part entière. Le mandat public officiel existe, mais il ne fait pas pour autant une profession. C'est vrai qu'il y a des associations de travailleurs sociaux (la culture 
professionnelle) et que des individus sont formés pour le travail social (la base théorique existe, mais sa solidité et son organisation pose d'autres problèmes). De plus, plusieurs dilettantes gravitent autour du champ social. Le code de déontologie n'a pas encore été adapté au contexte sénégalais et il pourrait faire l'objet d'un débat dans le cadre d'un service social international. Il faut retenir, en dernier lieu, que la grande majorité des travailleurs sociaux sont des fonctionnaires de l'État sénégalais.

L'inexistence d'un référentiel limite le déploiement du service social au Sénégal. Comme le dit si bien Nicole Ramogino (cité par d'Alverny 2000), il existe au fondement du social, une dimension symbolique qui rend possible et nécessaire toute pratique sociale dans sa réalité et sa réalisation. Au Sénégal, ce symbolisme n'a jamais existé comme contrat entre le service social et la société sénégalaise. Prenons un exemple, le processus de stigmatisation du pauvre. Ce processus, si courant dans les analyses de la pauvreté en Amérique du Nord, ne désigne aucune réalité, aucun fait dans la société sénégalaise: la figure du pauvre, avec ses stigmates, n'ayant aucune référence dans la société traditionnelle. En effet en Afrique en général, au Sénégal en particulier, la marginalisation et l'exclusion n'ont jamais été, contrairement à l'Occident, considérées comme des coûts nécessaires et non productifs liés au développement. Considérée la pauvreté sous cet angle est un luxe, comme le pensent certains experts, hors de la portée des pays africains où la pauvreté est généralisée.

Les axes devant former l'armature des lignes à venir seront constitués d'une présentation sommaire du Sénégal, suivi d'une discussion sur la place qu'occupe le service social dans la société sénégalaise à travers son histoire et ses transformations. Nous aborderons ensuite les éléments liés à la formation avant de conclure.

\section{Généralités sur le Sénégal}

Le Sénégal («sunugal», notre pirogue ou senghâna selon les historiens), dont la capitale fut celle de l'Afrique Occidentale 
Française (A.O.F), occupe une position de Finistère ouest du continent africain. Le pays à dominante de plaines, est limité à l'ouest par l'océan atlantique, à l'est par la République du Mali, au nord par la République islamique de la Mauritanie, au sud par la Guinée Bissau et la République de Guinée. Le climat est de type soudano-sahélien, avec des contrastes du sud au nord. Il est caractérisé par l'alternance de deux saisons principales: une pluvieuse (de juillet à septembre) et une sèche (d'octobre à juin). Traditionnellement, on peut noter l'existence de quatre saisons.

Des forêts subsistent au sud du pays, alors que le centre est occupé par une savane herbacée avec l'existence de graminées. Le nord qui flirte avec le désert, est aride. Les cours d'eau ne sont pas aussi omniprésents au Sénégal qu'au Canada.Trois principaux fleuves arrosent le pays (le fleuve Sénégal, le fleuve Gambie et le fleuve Casamance). Les lacs y sont peu nombreux (lac de Guiers, lac Retba et lac Tanma).

Le Sénégal compte actuellement dix circonscriptions administratives avec 35 habitants au $\mathrm{km}^{2}$. La capitale Dakar, presqu'île situé à l'extrême ouest du pays, compte 2770 habitants au km² avec seulement $0,3 \%$ de la superficie du pays. Elle polarise plus de 2800000 habitants sur les 9524089 que compte le pays. Le taux de croissance démographique est $2,7 \%$.

L'économie sénégalaise repose sur l'agriculture, les phosphates, la pêche et le tourisme. Dans ce contexte d'interdépendance et de globalisation, la photographie des déterminants sociaux internes du Sénégal donne l'image d'une société prise entre une tradition sécurisante et un «modernisme» mal digéré, comme l'a noté Boudreau (2000). Les mutations se produisent à une vitesse exponentielle. La crise, accentuée par la dévaluation du franc CFA, influe sur les comportements. Les valeurs de solidarité et le sens de la famille s'effritent. La pauvreté s'affiche partout malgré les signes épars de l'opulence agressive d'une minorité.

Au Sénégal $35 \%$ des ménages vivent sous le seuil de pauvreté tel que définit par les normes de l'O.M.S. (Cahiers africains du travail social 2000). Le PNB est de $510 \$$ par habitant. La pauvreté rurale semble plus structurelle que la pauvreté urbaine. 
Sur le plan politique, le Sénégal occupe une place de choix en Afrique avec son multipartisme, en vigueur depuis 1974, sa presse relativement libre, ses élections régulières. Son système de santé et d'éducation, l'un des plus anciens en Afrique, est performant, malgré des contraintes majeures.

\section{L'évolution du service social au Sénégal}

\section{Le service social avant l'indépendance}

Le service social colonial fut crée par la loi du 19 novembre 1943 et rattaché au ministère des colonies. À l'époque, le service social colonial avait un double objectif, soit de lutter contre les répercussions de la guerre sur les familles des colons et d'intervenir auprès des indigènes. En Afrique occidentale française et au Sénégal qui abritait sa capitale, le service colonial fut introduit en 1948. Ses missions furent élargies par Bernard Cornut Gentille en 1952 et 1955. Des travailleurs sociaux furent affectés en Afrique et opérés à partir du bureau des affaires sociales du Sénégal (Audibert 1977).

Les activités, relevant du bureau des affaires sociales, étaient limitées au tricotage, à la couture et à l'économie familiale. Cellesci étaient en décalage avec les besoins des usagers. D'autres services sociaux virent le jour pour répondre à des besoins spécifiques: service social de la PMI (protection maternelle et infantile), service d'hygiène scolaire pour l'enseignement, service social auprès du tribunal, service social des caisses d'allocation familiales.

Le modèle de service social implanté au Sénégal résulte de facteurs idéologiques et économiques. Sur le plan idéologique, ce modèle se nourrissait au «mythe du développement» en prônant des «changements de structures» capables de permettre une prise en charge des indigènes, semblable à celle qu'avaient connu les colons. Une sorte de «darwinisme social» avait guidé le décideur français, en instaurant le principe de la sélection des mieux adaptés. Sur le plan économique, il fallait concilier, comme l'a dit Audibert (1977), la nécessité de faire fonctionner les exploitations agricole 
et industrielle avec l'obligation de maintenir en ordre «la société indigène».

Ces facteurs montrent que l'émergence du service social au Sénégal ne relève ni d'une volonté de contrôle social des classes dangereuses ni d'une pression sociale issue d'une solidarité de classes, mais des contraintes de valorisation et reproduction coloniale (Mbodj 2000).Toutes les dispositions relatives à la sécurité de l'emploi et à l'assistance sociale ont été mises en place pour répondre au marché français.

Le modèle de service social, de ce point de vue, n'est pas opérationnel dans le contexte sénégalais. Les normes, les valeurs d'une société donnée ne peuvent pas se transférer de manière mécanique à une autre société. La logique d'accumulation pouvait difficilement se substituer à la logique distributive et communautaire.

Il faut signaler que la prise en charge de l'enfance délinquante évoluait dans l'ombre des missions traditionnelles du service social colonial. En effet, la première structure de prise en charge de l'enfance remonte à 1888. Il s'agissait de fermes écoles pénitentiaires. La perspective pénale de la prise en charge était en cours. Les châtiments corporels et le travail forcé prenaient le dessus sur l'éducation. Avec l'élargissement des missions du bureau des affaires sociales, des structures fermées de prise en charge de jeunes délinquants furent crées: centres d'accueil et d'observation de mineurs inadaptés, chantiers agricoles, etc. La perspective d'un service social s'esquissait de manière timide. La création du service social auprès du tribunal renforça le dispositif concernant la prise en charge des mineurs délinquants.

\section{Le service social après l'indépendance}

Comme nous venons de le montrer, l'avènement du service social au Sénégal est le fait de l'administration coloniale française, mais au cours des années 1960, celui-ci connut un développement autonome. Nous rappellerons ici les principaux repères historiques ayant jalonné ce développement ainsi que les axes majeurs d'une politique d'action sociale au Sénégal. 
En 1960, on assiste au transfert des compétences du bureau des affaires sociales avec son rattachement au nouveau ministère de la santé publique du Sénégal. On assiste à la création du service des affaires sociales à la place du bureau des affaires sociales aux ministères $\mathrm{du}$ travail et des affaires sociales. Puis, il y a une réorganisation du service des affaires sociales (décret 60-108 du 9 juillet 1960) sous le registre de l'assistanat par la création des centres sociaux dans les villes et autres zones à problèmes. Toujours par décret, on établit une organisation et une réglementation des secours (décret 60245 du 13 juillet, 1960) à travers l'aide d'urgence ponctuelle (monétaire ou en nature, denrées de première nécessité) à toute personne démunie qui en fait la demande. En 1966, un tribunal pour enfants et un service spécialisé pour la prise en charge des mineurs délinquants et ceux en danger moral ont été crées (décret 146 du 10 octobre 1966). En 1968, on assiste à la création d'une division des affaires sociales (décret 108 du 9 mars 1968) ayant comme attribution principale, l'étude des problèmes sociaux, le contrôle des institutions sociales, publiques et privées, la protection de la famille et de l'enfance, l'assistance aux handicapés et la prise en charge de l'enfance délinquante et en danger moral.En 1969, il $\mathrm{y}$ a la constitution d'une allocation d'entretien aux enfants mineurs indigents, orphelins de père ou abandonnés et pupilles de l'État.

L'année 1975 marque la transformation de la direction des affaires sociales en direction de l'action sociale par le décret 75-548 du 22 mai 1975. Cette réforme visait à réduire l'assistanat par la recherche de moyens et d'un contenu plus dynamique à l'action sociale. En 1977, on assiste à la création de la direction de l'éducation surveillée et de la protection sociale (décret 77-659 du 25 juin 1977). Elle se substitue au service spécialisé pour la prise en charge des mineurs. La création du ministère chargé de l'action sociale (décret 78-237 du 15 mars 1978) eut lieu en 1978 et la direction de l'action sociale devient l'organe essentiel de ce ministère.

En 1981, il y a réorganisation de la direction de l'éducation surveillée et de la protection sociale. Puis, en 1983, il y a la création du ministère du développement social à la suite de l'adoption du plan d'action de Lagos en 1978 (sommet de l'O.U.A.) où les chefs d'État ont considéré que les facteurs humains et sociaux 
sont déterminants et qu'ils ont été un peu occultés dans la première décennie de développement de leurs pays respectifs. Le ministère du développement social était le résultat de la fusion entre le ministère de l'action sociale et le secrétariat d'État à la promotion humaine. Cette fusion déclencha une demande tout azimut de travailleurs sociaux par d'autres ministères (santé, éducation nationale, jeunesse, etc.). Le service social scolaire s'est structuré au Sénégal dans ce contexte. En 1988, il y a renforcement des prérogatives du ministère du développement social (décret 881569 du 28 novembre 1988). Les centres sociaux laissèrent la place aux centres de protection et de réinsertion sociale (CPRS). En 1990, la Direction de l'Action Sociale est rattachée au ministère de la santé publique et de l'action sociale avec la disparition du ministère du développement social. Ses missions n'avaient pas changé, mais la perspective médico-sociale gagnait du terrain.

De 1990 jusqu'en 2001, la Direction de l'action sociale valsa de tutelle en tutelle. Il y a eu après le ministère de la santé et de l'action sociale celle de la femme, de l'enfant et de la famille, puis celle de la famille et de l'action sociale et de la solidarité nationale, puis retour au développement social et de la solidarité nationale en attendant un autre remaniement ministériel ou un autre régime.

La Direction de l'éducation surveillée et de la protection sociale, quant à elle, resta inamovible, au Ministère de la justice. La timide croisade des travailleurs sociaux sur la déjudiciarisation n'y changea rien. Cet autre segment de l'action sociale au Sénégal évolua en catimini dans sa recherche de solutions pour mieux éduquer, rééduquer, insérer, réinsérer les jeunes délinquants.

\section{Les axes de la politique d'action sociale}

Les axes majeurs de la politique d'action sociale ont été l'assistance aux personnes déshéritées, l'insertion sociale des personnes handicapées, une politique de prophylaxie sociale (élaboration et mis en œuvre du programme d'information, éducation, prévention 
et communication), un programme en faveur de l'enfance en situation difficile, l'insertion des personnes du $3^{\text {ème }}$ âge, la coordination des actions sociales de certains ministères, organismes nationaux et internationaux. Cela dit, on n'a pas abandonné pour autant la prise en charge de l'enfance délinquante, le secours et l'aide sociale aux sinistrés, l'aide sociale aux pupilles de l'État. Cette politique d'action sociale comportait l'attribution de subventions aux institutions d'éducation non conventionnelles et aux «daaras» (écoles coraniques) ainsi que l'aide pour l'achat d'appareils pour les personnes handicapées (handicapées moteurs, visuels ou auditifs).

Les ressources humaines devant mettre en pratique ces différents axes de la politique sont, pour l'essentiel, composées de travailleurs sociaux. La gestion de l'identité socioprofessionnelle des travailleurs sociaux en France est en général peu pertinente, car politique. En revanche, celle des travailleurs sociaux sénégalais paraît à première vue simple. Par travailleurs sociaux, il est fait référence aux assistants sociaux, aux éducateurs spécialisés et aux aides sociaux (dans certains pays africains, on parle de conseillers aux affaires sociales, d'éducateurs sociaux, etc.). Au Sénégal, le travailleur social, même si la profession tarde à s'imposer, est clairement identifié alors qu'en France, on associe aux travailleurs sociaux, un premier groupe de sept professions sociales (assistants sociaux, éducateurs spécialisés, éducateurs de justice, conseiller en économie familiale, animateurs socioculturels, délégués à la tutelle, éducateurs spécialisés de jeunes enfants) et un deuxième groupe de cinq autres professions qui ont un rôle complémentaire.

Malgré la réception, le service social au Sénégal a évité de s'embarrasser de professions qui risquent de «miner» le social. D'autres professions se réclament du travail social, mais l'État sénégalais (la fonction publique) ne les a pas encore reconnues, comme il n'a pas adopté le concept de travailleurs sociaux. Les éducateurs spécialisés, les assistants et aides sociaux ont reçu chacun, un statut particulier de fonctionnaires du ministère de tutelle respectif.

Un personnel technique et administratif accompagne les travailleurs sociaux dans leurs tâches quotidiennes (moniteurs techniques, maîtresse d'économie familiale, enseignants, etc.). 


\section{Les structures ou agences sociales}

Le service social au Sénégal se développe plus à partir des structures étatiques même si les structures privées sont présentes dans le champ. L'essentiel des travailleurs sociaux évolue dans ces structures. L'arrêt du recrutement dans la fonction publique a facilité le déploiement des nouveaux diplômés des écoles de formation vers d'autres zones de travail. Les deux structures étatiques où gravitent le plus de travailleurs sociaux sont:

1) la Direction de l'Action Sociale (D.A.S.) qui disposent de services centraux et de structures de base. Elle est actuellement sous la tutelle du Ministère du développement social et de la solidarité nationale. La division des centres sociaux et de la prophylaxie sociale, celle des secours et d'aides, celle de la promotion des personnes handicapées sont ses principaux démembrements au niveau central;

2) les centres de promotion et de la réinsertion sociale (C.P.R.S., les ex-centres sociaux) constituent, avec les coordinations régionales de l'action sociale, les structures de base.Les C.P.R.S., disséminés dans chaque région du pays ont comme activités principales, l'alphabétisation, l'art ménager, la puériculture, l'I.E.C. (Information, Éducation, Communication) en matière de santé, de lutte contre la pauvreté (appui au micro-projet). Les jeunes filles et les femmes en général sont leurs cibles traditionnelles, même si des personnes de toutes catégories sociales les sollicitent. Ils perpétuent essentiellement dans leurs fonctions, les orientations du service social colonial.

La Direction de l'Éducation Surveillée et de la Protection Sociale (D.E.S.P.S.) dispose également de services centraux et des structures extérieures. Placée sous la tutelle du Ministère de la justice depuis sa création, elle comprend: une division administrative financière qui s'occupe des infrastructures et de la logistique; une division de l'action éducative et de la protection sociale (D.A.E.P.S.) qui centralise les enquêtes sociales; une division des études de la recherche, des statistiques et de la formation (D.E.R.S.F.). Ces 
divisions sont structurées en bureaux pour en faciliter le fonctionnement.

Les structures extérieures ont presque toutes pour mandat la prise en charge de l'enfance délinquante et en danger moral, à travers des activités techniques et psychopédagogiques appropriées. Les structures sont fermées (centres d'adaptation sociale), semifermées (centre de sauvegarde), ouvertes (action éducative en milieu ouvert) ou polyvalentes.

Les missions des trois premières structures tournent principalement autour de l'enseignement, de l'ergothérapie et des activités socio-éducatives. Le service de l'action éducative en milieu ouvert s'occupe pour sa part de la postcure d'internat, c'est-à-dire de la réinsertion socioprofessionnelle du jeune après un séjour en milieu fermé, des placements scolaires et professionnels, d'enquêtes de divorce, d'adoption. Dans chaque région, il y a un service A.E.M.O. qui travaille en étroite collaboration avec un tribunal pour enfants.

Le Sénégal compte 113 ans de prise en charge de mineurs à comportement problématique. Certaines structures ont développé un partenariat et continuent de tisser des liens avec des structures françaises. Il existe aussi au Sénégal des structures à vocation sociale, gérées par d'autres ministères ou des organismes nationaux et internationaux. Il faut aussi noter l'existence d'un fonds national d'action sociale, logé à la Présidence de la république, qui accompagne certaines institutions luttant contre l'inadaptation et la pauvreté.

Les travailleurs sociaux, en dehors des structures, évoluent aussi dans des associations. L'A.N.A.S.S.A.S. (Association nationale des assistants et aides sociaux) et l'A.E.S. (Association des éducateurs spécialisés) sont les plus anciennes et les plus représentatives. D'autres réseaux ont vu le jour comme le R.N.T.S. (Réseau national des travailleurs sociaux), le R.A.T.S. (Réseau africain des travailleurs sociaux) ou l'O.P.T.S. (Organisation panafricaine des travailleurs sociaux). Ces associations se sont créées à partir de la nécessité de faire reconnaitre la profession de travailleur social. Depuis l'échec de l'A.N.T.S. (Association nationale des travailleurs 
sociaux), il faut dire que les professions sociales même si elles ont les mêmes objectifs, ne marchent pas toujours ensemble afin de les atteindre.

\section{La formation des travailleurs sociaux au Sénégal}

\section{L'École Nationale des Assistants Sociaux et Éducateurs Spécialisés (ÉNAES)}

L'enseignement moderne du service social a débuté au Sénégal en 1969 avec la création de l'A.N.A.E.S. (École nationale des assistants sociaux et éducateurs spécialisés). Auparavant, la formation des travailleurs sociaux s'effectuait en France, à l'Université d'Aix-en-Provence. L'E.N.A.E.S fut la première école de formation de travail social au Sénégal et en Afrique, au sud du sahara. L'expertise pour la formation des éducateurs était au début française et israélienne. L'E.N.A.E.S. formait au premier cycle, pour trois années, des détenteurs de toutes nationalités de baccalauréat ou l'équivalent, à l'exception des aides sociaux. Plusieurs générations de travailleurs sociaux africains (plus de quinze nationalités) y ont été formés et cette formation a été démultipliée.

L'École formait donc, comme professions sociales, des éducateurs spécialisés et des assistants sociaux en deux années de tronc commun et une de spécialisation. La perspective médicosociale était omniprésente dans la formation. Les programmes s'articulaient autour des disciplines suivantes: la méthodologie d'intervention en service social (service social personnel, service social avec les groupes, service social communautaire), aux disciplines liées à la santé (protection maternelle et infantile, nutrition, santé maternelle et infantile, hygiène, pathologie et psychiatrie), aux disciplines liées au droit (droit civil, droit social, droit pénal), à la méthodologie de la recherche et la sociologie, aux disciplines liées à la psychologie (psychologie génétique, générale, sociale, psychopathologie et psychopédagogie), à la 
démographie et aux statistiques, à la géographie politique et économique, à l'économie, aux techniques professionnelles.

Un diplôme d'État était délivré à la fin du cursus (Diplôme d'État d'éducateurs spécialisés, Diplôme d'État d'assistant social). Tous les diplômés sénégalais étaient recrutés par l'État (cinq par année). Grâce à la coopération française (envoi de professeurs permanents, subventions, etc.), l'E.N.A.E.S. a su relever le défi de la formation des cadres moyens pour le service social africain. Elle avait développé un partenariat fécond avec l'Université d'Aixen-Provence, en France, puis avec l'Université Laval au Canada pour la formation des formateurs.

Le programme de restructuration des écoles de formation, entamé en 1993, suite aux injonctions de la banque mondiale, n'épargna pas l'E.N.A.E.S. Une partie de son effectif (assistants et aides sociaux) fut transférée dans une nouvelle école de service social et de santé, crée sur les cendres de l'école des infirmiers d'État. La création des nouvelles filières sauva l'effectif d'étudiants restant à l'E.N.A.E.S. (éducateurs spécialisés) et une nouvelle école de service social vit le jour.

\section{L'École Nationale des Travailleurs Sociaux Spécialisés}

L'École Nationale des travailleurs sociaux spécialisés (E.N.T.S.S.) est née des cendres de la défunte E.N.A.E.S. Sa mission s'inscrit dans le décret 94-562 du 02 juin 1994 portant sur la création et l'organisation de E.N.T.S.S. et précise à son article premier que l'école a pour vocation principale la formation des cadres supérieurs dans le domaine du travail social spécialisé, la formation permanente des travailleurs sociaux et la recherche scientifique dans le domaine du travail social.

À partir de cette mission, ont été définis les axes pédagogiques suivants: mise en exergue de l'enseignement des modalités d'intervention et des pratiques en travail social et de la recherche, qui constitue le noyau dur de la formation; identification et la réactualisation des enseignements spéciaux de manière à permettre à l'étudiant de maîtriser son futur champ d'intervention; assurer 
la formation d'agents opérationnels par l'alternance école-terrain; l'évaluation des actes de formation; le renforcement du taux d'encadrement et celui de la recherche et de la formation permanente; l'accès des étudiants à toutes les innovations technologiques.

\section{Organisation pédagogique de l'É.N.T.S.S}

\section{Enseignements théoriques}

Ils sont structurés autour de trois niveaux pour le premier et le second cycle. Le noyau dur de la formation en travail social et en recherche comprend: les trois dimensions de l'intervention et de l'analyse en travail social, la politique sociale, la supervision, l'introduction au service social et le cadre théorique, les politiques publiques. La recherche comprenant le processus de recherche pour le premier cycle et toutes les techniques et méthodes de recherches pour le second cycle (640 heures).

\section{Enseignements spéciaux}

Il s'agit de toutes les connaissances spécifiques, méthodes et techniques utiles à la méthodologie du champ d'intervention de l'étudiant. L'amplitude des disciplines varie en fonction de l'adéquation de la formation en emploi. Par exemple, au premier cycle, les principaux enseignements spéciaux par filières sont:

1) filière éducation surveillée: déviance et délinquance, droit, santé mentale, gestion des projets, techniques professionnelles, psychopathologie, psychopédagogie;

2) filière travail social communautaire: politique de population, économie du développement, gestion des collectivités locales, production animale et végétale, comptabilité, projets;

3) filière travail social d'entreprise: management, marketing, droit du travail et des affaires, sociologie des organisations, gestion des ressources humaines, gestion financière, comptabilité; 
4) filière formation et réinsertion des personnes handicapées: psychologie, réadaptation, langage des signes, braille, orthophonie, psychopathologie, projets.

Au deuxième cycle, les principaux enseignements spéciaux par filières sont:

1) filière gestion des services sociaux et de l'éducation surveillée: marketing, management, politique publique, gestion financière, comptabilité analytique, droit administratif, droit social, droit civil, statistiques, anglais des affaires;

2) filière formation des formateurs: La formation des formateurs comprend l'animation pédagogique, théorie de l'éducation, statistique de l'éducation, planification, psychopédagogie, droit (civil, administratif et social), déviance et délinquance.

\section{Éclairages théoriques}

Il s'agit de cours choisis en fonction de leur pertinence dans la formation générale des travailleurs sociaux et qui contribuent à l'éclairage des champs d'interventions. Les enseignements théoriques occupent un volume horaire de 1600 heures au premier cycle. Il s'agit de la sociologie, de la gestion de l'environnement, de la psychologie du développement humain, de la démographie, de la psychologie générale, de l'informatique. Les enseignements théoriques au second cycle sont organisés sous forme de modules et séminaires d'enseignement, cotés sur la base d'un crédit pour 45 heures d'études. Les étudiants pourront demander la validation de crédits pour des modules déjà reçus ailleurs.

\section{Stages}

Ils occupent 1400 heures dans le modèle pédagogique. On distingue trois sortes de stage: 1) les stages exploratoires (milieu urbain et milieu rural), 2) les stages d'imprégnation et d'analyse (dans les institutions de prise en charge ou agences sociales) et, 3) les stages très professionnels. Les étudiants du $2^{\mathrm{e}}$ cycle effectuent en plus un stage d'application. 


\section{Modalités d'enseignement}

L'enseignement d'une discipline à l'E.N.T.S.S. est conditionné par l'acceptation d'un syllabus qui respecte les objectifs de formation fixés par l'école. Les professeurs déposent leur syllabus auprès des chefs de départements qui les transmettent après étude et avis au directeur des études et des stages. Le syllabus est validé éventuellement par le conseil de direction qui peut commettre son consultant spécialisé dans la discipline en l'absence d'un professeur responsable du module. Le syllabus retenu est négocié, pour ce qui concerne le mode d'évaluation avec les étudiants, sous réserve de dispositions prévues par l'arrêté n 07142 du 27 août 1994. Le nombre d'heures d'enseignement et les modalités sont fixés par le conseil de direction. Les professeurs sont évalués tant en ce qui concerne le contenu des enseignements que la méthode pédagogique par les élèves chaque trimestre ou à la fin de chaque module de formation. Une séance de restitution peut être programmée par la direction des études et stages ou le chef de département avec le professeur intéressé.

\section{Le système d'évaluation}

Le système d'évaluation varie d'un cycle à l'autre. Au second cycle, les cours suivis avec succès donnent droit au nombre de crédits prévus par le programme. Le crédit représente quarante cinq heures de travail consacrées par l'élève à une activité (cours, travaux de recherche). Les crédits peuvent être obtenus par validation suite à une requête introduite par l'étudiant auprès du chef de département, si l'élève a déjà fait le cours ou un cours équivalent. Les crédits sont validés après avis motivé du chef de département et du directeur des études et des stages par le conseil pédagogique.

L'évaluation d'un cours donne droit à une valeur numérique allant de 5 à 0 et le nombre de crédits prévus est obtenu par l'étudiant si et seulement si la note est égale ou supérieure à 2 (égale ou supérieure à 12 dans le système de notation français). Les élèves peuvent être inscrits à temps complet ou en temps partiel, mais dans les deux cas, ils obtiennent le diplôme supérieur 
en travail social s'ils réunissent les trois exigences suivantes:

1) une note de scolarité (56 crédits),

2) une note de soutenance de mémoire (6 crédits) et,

3) une note d'examen de fin d'études (6 crédits). Un élève ne peut se présenter à l'examen de fin d'études s'il n'a pas réussi les 56 crédits prévus.

Les modules dispensés au niveau du département de la formation permanente et de la recherche peuvent donner droit à des crédits de programme s'ils respectent les conditions fixées par le programme du DSTS.

Au premier cycle, les cours sont évalués de 0 à 20 et sont cotés suivant leur importance dans la formation du travailleur social, leur pertinence quant à la filière de formation ou leur capacité d'éclairage théorique. Les coefficients varient en fonction de l'appartenance d'un cours à un palier ou un autre du programme.

Les enseignements du noyau dur dans la formation des travailleurs sociaux sont cotés de coefficient 4, les enseignements spéciaux sont cotés de coefficient 3, les cours d'éclairage théorique sont cotés de coefficient 2 . Les stages sont cotés de coefficient 3 quelle que soit la filière. La note de passage en classe supérieure et celle de certification est de 12 .

Les élèves du premier cycle en fin de formation obtiennent le diplôme d'état de travailleur social sur la base des trois notes suivantes:

1) la note des trois années de scolarité ayant un coefficient de 5 ,

2) la note de mémoire de fin d'études, un coefficient de 4 et,

3) la note de l'examen de fin d'études, un coefficient de 6.

L'É.N.T.S.S. dispose d'un département de formation permanente et de recherche qui s'occupe de l'animation institutionnelle (organisation des conférences, symposiums, etc.) en plus de sa mission première (recherche et formation permanente). Il s'occupe également de la gestion technique et administrative des mémoires de fin de cycle, de même que la revue de l'institution (Cahiers africains du travail social). L'E.N.T.S.S., comme la plupart des écoles de service social, entreprend de 
nouvelles initiatives (en sus de celles de l'E.N.A.E.S.) pour répondre à l'interdépendance globale. Plus largement, il s'agit d'un défi à relever par le service social sur le plan national et international. Le salut, à notre point de vue, réside dans cette perspective, car même si le contenu s'enracine au plan national, il y a certains points focaux partagés par tous: droits de la personne, développement social et compétence culturelle. C'est dans ce cadre que l'E.N.T.S.S. pense élargir ses partenaires. Le projet de coopération avec l'École de service social de l'Université Laurentienne se situe dans cette nouvelle orientation.

L'École Nationale de Développement Social st Sanitaire (É.N.D.S.S):l'ancienne école nationale des infirmiers et infirmières d'état du Sénégal a vu, dans le cadre de la restructuration des écoles nationales de formation (1993), sa mission élargie. Elle accueille en plus de ses filières traditionnelles (infirmiers, infirmières et sage femmes) la filière des agents d'hygiène, des aides et assistants sociaux (la première promotion venait de l'E.N.A.E.S.), des techniciens supérieurs de la santé. L'E.N.D.S.S est une institution de formation à vocation sociale et sanitaire.

Pour l'essentiel, ses programmes en direction des futurs travailleurs sociaux s'inspirent de ceux de l'E.N.A.E.S., car elle a accueilli les assistants et aides sociaux) et le programme de la défunte école. Dans le cas des assistants sociaux, elle les forme, comme à l'E.N.A.E.S. pour trois années (après le baccalauréat) en vue de l'obtention du diplôme d'état d'assistant social (D.E.A.S.). La perspective médico-sociale se voit ainsi perpétuée par l'E.N.D.S.S. alors que l'E.N.T.S.S. se «cramponne» au service social stricto sensu.

Nous signalons que les sortants de l'E.N.E.A. (École Nationale d'Économie Appliquée) s'assimilent à des travailleurs sociaux (collège d'animation).

L'approche de la formation des travailleurs sociaux peut rebuter, tant l'ensemble donne l'impression d'un véritable capharnaüm. Les disciplines enseignées font légion, mais certaines d'entre elles n'ont pas leur place dans les dispositifs pédagogiques. De plus, les écoles de service social au Sénégal sont coupées du monde 
universitaire et n'ont pas comme les écoles de service social occidentales, une tradition d'échange et de collaboration. Les départements de sociologie des universités qui sont censés abriter ces écoles les snobent. Ainsi, les programmes occultent la composition sociale, la tradition culturelle et l'environnement organisationnel.

\section{Conclusion}

Force est de reconnaitre que le service social au Sénégal repose sur des textes peu adaptés au contexte. La mission est mal définie, voire floue, ses gérants travaillent dans la dispersion, ses approches sont inadaptées. En explorant le champ du service social au Sénégal, le lecteur peut avoir une impression de complexité.

Le service social au Sénégal parait être un territoire inconnu aux frontières du social, du sanitaire, du juridique. La question de l'identité professionnelle pose problème de même que les mécanismes de résolution des problèmes sociaux et la vocation des structures d'assistance. Le concept de travailleur social n'est pas encore adopté par les pouvoirs publics et la population.

L'introduction du service social dans un pays sous-développé comme le Sénégal signifie qu'il faut aborder les questions qui dépassent les rapports politiques. Son développement s'est fait, comme nous l'avons montré plus haut, dans la foulée de l'applicabilité universelle de la supériorité du modèle occidental. Les pressions exercées par les changements démographiques, socioculturels, économiques et politiques, remodèlent les milieux où se pratique le service social. Les pratiques sociales doivent être bien enracinées dans leur contexte.

Pour assurer le développement du service social au Sénégal, il faut impérativement aborder les problèmes conceptuels fondamentaux et tenir compte du discours idéologique, épistémologique et technique car le service social est un produit socioculturel qui prend racine dans ces discours. La réponse face 
à la dérégulation sociale qui s'est opérée en Afrique, au Sénégal, n'est pas tributaire, selon nous, d'une réponse instrumentale et fonctionnelle d'une société face à ses problèmes.

Le discours professionnel n'a jamais été construit à partir des écoles de service social. Au plan de la demande sociale initiale, le modèle français d'intervention s'avère peu efficace car le service social au Sénégal se doit d'être le construit de solidarités endogènes. L'émergence d'un modèle indigène n'a jamais été envisagée dans le temps ni dans l'espace discursif qui doit le supporter.

\section{Bibliographie}

AUDIBERT,André (1977), «Le service social francophone dans une perspective de développement», Thèse I et II, PRS Université PRS Sorbonne.

BEAUVOLSK, M. A. et al. (2001), Introduction à la pratique du service social, Sudbury, Université Laurentienne.

BOUDREAU, F. (2000), «Entre le néolibéralisme et le bien-être social: essai sur le développement et la culture au Sénégal», Cahiers africains du travail social, no 1, janvier, 63-91.

COLLECTIF (1996), "Actes du symposium sur la politique sociale au Sénégal», Cahiers africains du travail social, Dakar, Sénégal.

D'ALVERNY, Michel (2000), «De la reproduction du modèle du travail social à la française à l'émergence d'une politique sociale alternance au Sénégal», Cahiers africains du travail social, Dakar, Sénégal.

DIRECTION DE LA PLANIFICATION DES RESSOURCES HUMAINES (2000), «Stratégie 2000», Dakar, Sénégal.

MBODJ, M. (2000), «Des structures de solidarité à l'émergence d'une politique sociale alternative au Sénégal», Cahiers africains de travail social, École national des travailleurs sociaux (ENTSS), Dakar, 69-76.

SÉMINAIRE D'ÉVALUATION DES PROGRAMMES (1996), «Le modèle pédagogique de L'ENTSS», Dakar, Sénégal, 10-78.

UNICEF (2001), «Rapport 200», Dakar, Sénégal. 